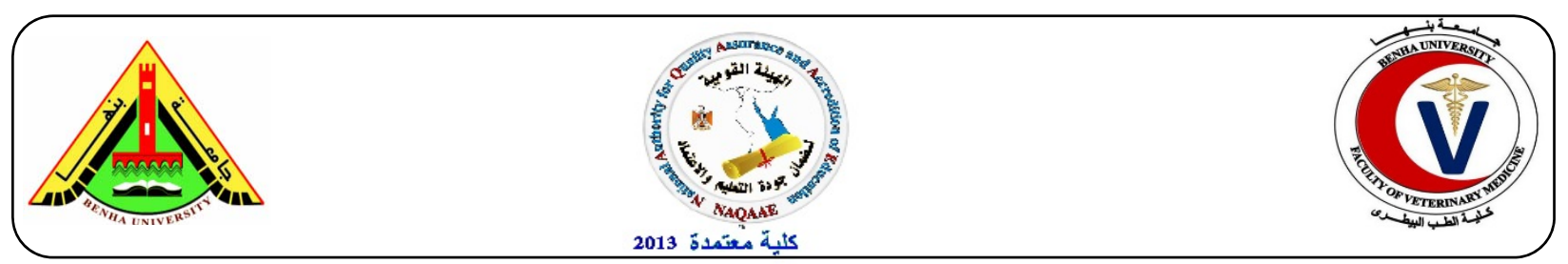

\title{
Clinical, hematobiochemical and histopathological changes in goats with surgically- induced unilateral Hydronephrosis.
}

\author{
Ghanem, M. M. ${ }^{1}$, Hayat Fayed ${ }^{1}$, Abdel-Raof, Y. M. ${ }^{1}$, El-Attar, H. M. ${ }^{1}$ and Al-Akraa, A. M. ${ }^{2}$ \\ ${ }^{\prime}$ Department of animal medicine ‘ Faculty of Veterinary Medicine ‘ Benha University ‘ Egypt. \\ ${ }^{2}$ Department of veterinary surgery, Faculty of Veterinary Medicine, Benha University, Egypt. \\ MOHAMED.GHANEM@fvtm.bu.edu.eg
}

\begin{abstract}
A B S T R A C T
This study aimed to evaluate the clinical, haematobiochemical and histopathological changes in goats with surgically induced unilateral hydronephrosis. To achieve this aim eight male castrated goats were divided into two groups. Group A (animals with surgically-induced unilateral hydronephrosis) in which goats were subjected to ligation of the right ureter for 42 days and group B (sham-operated). Clinical examination and haematobiochemical analysis were performed before and after induction (on days 1, 7, 14, 21, 28, 35 and 42). Histopathological examination of kidneys was carried out at the end of the study. On the $1^{\text {st }}$ day goats with unilateral hydronephrosis showed depression, arched back, decreased appetite. After that, these clinical signs were ameliorated and the animals restored their apparent health condition. $\mathrm{PCV} \%, \mathrm{Hb}$ content and RBCs count increased during the early periods of the study then returned gradually to normal level by the end of the study. WBCs count was significantly increased allover the time of the study. Significant increase of serum urea, creatinine, uric acid and calcium levels during the early period of the study then gradually decrease. Albumin and total proteins levels were initially decreased but were restored by the end of the experiment. Post-mortem examination of affected kidneys showed dilatation of renal pelvis and calyces with severe atrophy of the parenchyma. Histopathological examination of affected kidneys revealed severe cystic dilatation of renal tubules and intertubular connective tissue proliferation. We concluded that unilateral hydronephrosis produced significant changes during the early stage, which were gradually restored owing to the compensatory activities of the contralateral kidney.
\end{abstract}

Keywords: Ureter, ligation, hydronephrosis, kidney, goats.

(http://www.bvmj.bu.edu.eg)

(BVMJ-28(2): 263-272, 2015)

\section{INTRODUCTION}

$\mathrm{T}$ The principal function of urinary system is maintenance of water and electrolytes homeostasis, which require that any input into system be balanced by an equivalent output. The urinary system provides the mechanism by which excess water and electrolytes are eliminated from the body. The second major function of this system is the excretion of many toxic metabolic waste products particularly the nitrogenous compounds urea and creatinine. The affection of the urinary system, thus responsible for the alteration of excretion of waste products from body which disturb homeostasis; this not only causes damage to the organs of the urinary system but also affects whole body. Selective reabsorption and conservation of useful substances, e.g. Glucose and sodium chloride also occur in the kidney (Chauchan, 1995). Diseases of the kidneys, and in some instances of the ureters, bladder, and urethra, reduce the efficiency of the kidney's functions, resulting in disturbances in protein, acidbase, solute and water homeostasis and in the excretion of metabolic end-products. A partial loss of function is described as renal 
insufficiency. When the kidneys can no longer regulate body fluid and solute composition, renal failure occurs (Radostits et al., 2007). Hydronephrosis is a disease that occurs due to complete or partial obstruction of the urinary flow. This causes progressive distension of the renal pelvis and diverticula, which results in atrophy of the renal parenchyma and loss of function. The severity of the lesion depends on the time elapsed since the onset of obstruction (Santarosa et al., 2007). Therefore, this work aimed to evaluate clinical, hematobiochemical and histopathological changes resulting from surgical induction of unilateral hydronephrosis in goats.

\section{MATERIALS AND METHODS}

\subsection{Experimental animals}

A total number of eight clinically healthy adult castrated male goats were used in the experiment. Their age ranged from 1-1.5 years and weight about 16-24 Kg. The experimental animals were de-warmed with systemic anthelmintic Ivermectin + Clorsulon $0.2 \mathrm{mg} / \mathrm{kg}$ Bwt. (Ivomac $\AA$ super in a dose of $1 \mathrm{ml} / 50 \mathrm{~kg} \mathrm{~S} / \mathrm{C}$ ). The animals were fed on green fodder and concentrate with free access to sufficient tap water and placed good hygienically well ventilated room and kept under the same environmental, nutritional and hygienic conditions throughout the period of the experiment. The animals were left for 2 weeks for acclimatization before the beginning of the experiment. During this period, they were subjected to a clinical investigation to ensure that they are clinically healthy.

\subsection{Animal grouping and experimental design}

Eight castrated male goats were randomized divided into 2 groups: In group $\mathrm{A}$ : (surgically-induced unilateral hydronephrosis) five goats that were subjected to experimentally-induced unilateral Hydronephrosis by ligation of the right ureter as previously described by
Khalil (2012) .Group B: 3 goats were subjected to the same surgical manipulation without ligation of the ureter as previously conducted by Khalil (2012). The goats in the two groups were evaluated by clinical examination and laboratory analysis before surgery and on the $1^{\text {st }}, 7^{\text {th }}, 14^{\text {th }}, 21^{\text {th }}, 28^{\text {th }}$, $35^{\text {th }}$ and $42^{\text {th }}$ days after surgery.

\subsection{Clinical examination:}

Observation and recording of clinical signs of goats after induction of hydronephrosis was conducted as described by Radostits et al. (2007).

\subsubsection{Samples:}

Blood samples were collected from jugular vein according to Radostits et al. (2007) to be used for hematological examination. In addition, clear non haemolyzed sera were separated for biochemical determination of urea, uric acid, creatinine, albumin, globulin, total protein, sodium, potassium, chloride, calcium and phosphorus.

\subsubsection{Haematological examination}

The total number of erythrocytes per cubic millimeter, the total number of leukocytes per cubic millimeter, the hemoglobin content and the $\mathrm{PCV} \%$ were determined according to Bernard et al. (2000).

\subsubsection{Biochemical analysis of serum}

The serum samples were used for spectrophotometric determination of urea (Patton and Crouch, 1977), uric acid (Wilding and Heath, 1975), creatinine (Young, 1990), albumin (Fischbach and Dunning, 2009), total proteins (Pagana and Pagana, 2010), potassium and sodium levels (Henry et al., 1974), calcium (Teitz, 1970), phosphorus (Anderson and Cockayne, 1993) and Chloride (Kaplan and Pesce, 1996).

\subsubsection{Gross examination of kidneys of necropsied goats}

Kidneys of each necropsied goat were carefully examined by naked eye for detection of any gross lesions of the examined 
kidneys according to Hall (1983).

\subsubsection{Histopathological examination}

Specimens from kidneys of about $0.5 \mathrm{~cm}$ thickness were collected immediately after scarification in $10 \%$ neutral buffered formalin and used for histopathological examination. Samples were taken and were prepared for histological examination according to Newman, et al. (2007).

\subsubsection{Statistical analysis}

The data were statically analyzed using One Way Repeated Measures Analysis of Variance (ANOVA) and independentsamples $\mathrm{T}$ test in the experimental study as previously described by Bailey (2008). We used SPSS version 16 software to conduct this analysis. Values were represented as means \pm standard error (SE). All differences were considered significantly among groups of the experimental study when $P \leq$ 0.05 .

\section{RESULTS}

\subsection{Changes in clinical parameters}

Goats with surgically-induced unilateral hydronephrosis showed depression, dullness, arched back and decrease in appetite than normal during the first day post obstruction. Moreover, pulse and respiration rates were significantly increased one day post-operative. Starting from the $3^{\text {rd }}$ day the goats restored their normal appetite, pulse and respiration rates. The temperature was not significantly affected by surgically-induced hydronephrosis all over the time of the study (Figure 1).

\subsection{Hematological changes}

The PCV\% was gradually decreased starting from the $1^{\text {st }}$ day and became significantly decreased on the $14^{\text {th }}$ day postoperative in goats with unilateral hydronephrosis. On day $21^{\text {st }}$ the PCV\% started to increase gradually till day $42^{\text {nd }}$ (Table 1). The $\mathrm{Hb}$ content showed a significant increase from the $7^{\text {th }}$ day postoperative and became highly significant increased on the $14^{\text {th }}$ and $21^{\text {st }}$ day then significantly decreased gradually until returned to its normal value at the end of the study (Table 1). The RBCs count showed non significant decrease in the first seven days followed by gradual increase until returned to its normal level on the $42^{\text {nd }}$ day (Table 1). The WBCs count was highly significantly increased on the $7^{\text {th }}$ day postoperative in control sham-operated goats then decreased gradually to reach its minimal value on the $42^{\text {nd }}$ day postoperative. The WBCs count was significantly increased from the $7^{\text {th }}$ day post-operative in goats with unilateral hydronephrosis and this increase remained until reached its maximal value on the $42^{\text {nd }}$ day post-operative (Table 1).

\subsection{Biochemical changes}

The serum urea level was significantly increased on the $7^{\text {th }}$ day post-operative then gradually decreased until reached nearly its normal level on the $42^{\text {nd }}$ day of the experiment (Table 2). The serum creatinine level showed significant increase on the $1^{\text {st }}$ day post-operative and the increase became significant on the $7^{\text {th }}$ day post-operative then gradually decreased until the end of the study (Table 2). The serum uric acid level revealed significant increased on the $7^{\text {th }}$ and $14^{\text {th }}$ day post-operative then significantly decreased until reached to the normal level at the end of the study (Table 2). The serum albumin and total protein levels were significant decreased on the $14^{\text {th }}$ day postoperative then gradually increased until reached near normal level on the $42^{\text {nd }}$ of the study (Table 2). The serum levels of sodium, potassium, chloride and phosphorus were insignificantly decreased and increased throughout the study (Table 2). The serum calcium level was significantly decreased on the $7^{\text {th }}$ day of the study and then gradually increased until reached the normal level at the end of the study (Table 2). 
Table (1): Hematological parameters of sham-operated goats and goats with surgically induced unilateral hydronephrosis (Means \pm S.E.).

\begin{tabular}{|c|c|c|c|c|c|c|c|c|c|c|c|c|c|c|c|c|}
\hline \multirow{2}{*}{$\begin{array}{c}\text { Days } \\
\text { Parameters }\end{array}$} & \multicolumn{2}{|c|}{0 day } & \multicolumn{2}{|c|}{$1^{\text {st }}$ day } & \multicolumn{2}{|c|}{$7^{\text {th }}$ day } & \multicolumn{2}{|c|}{$14^{\text {th }}$ day } & \multicolumn{2}{|c|}{$21^{\text {st }}$ day } & \multicolumn{2}{|c|}{$28^{\text {th }}$ day } & \multicolumn{2}{|c|}{$35^{\text {th }}$ day } & \multicolumn{2}{|c|}{$42^{\text {nd }}$ day } \\
\hline & Sh-o & $\mathrm{Hn}$ & Sh-o & $\mathrm{Hn}$ & Sh-o & $\mathrm{Hn}$ & Sh-o & $\mathrm{Hn}$ & Sh-o & $\mathrm{Hn}$ & Sh-o & $\mathrm{Hn}$ & Sh-o & $\mathrm{Hn}$ & Sh-o & $\mathrm{Hn}$ \\
\hline \multirow[t]{2}{*}{ PCV\% } & 27.67 & $28.63 \pm 0$ & $28 \pm 0.5$ & $27.77 \pm 0$ & $27.6 \pm 0$ & $26.67 \pm 0$ & $27.67 \pm$ & $25.33 \pm 0$ & $27.93 \pm$ & $27 \pm 0.58$ & $27.43 \pm$ & $27.73 \pm 0$ & $27.83 \pm$ & $28.67 \pm 0$ & $28.23 \pm$ & $28.83 \pm 0$ \\
\hline & \pm 0.73 & $.68^{\mathrm{b}}$ & 8 & $.62^{\mathrm{b}}$ & .62 & $.67^{\mathrm{b}}$ & 1.20 & $.88^{\mathrm{a}}$ & 0.30 & $\mathrm{~b}$ & 0.58 & $.67^{b}$ & 0.60 & $.88^{\mathrm{b}}$ & 0.48 & $.60^{\mathrm{b}}$ \\
\hline \multirow[t]{2}{*}{$\mathrm{Hb} \mathrm{g} / \mathrm{dl}$} & $10.8 \pm$ & $8.9 \pm 0.1^{\mathrm{c}}$ & $10.2 \pm 0$ & $9.5 \pm 0.2$ & $10.4 \pm 0$ & $9.97 \pm 0$. & $10.4 \pm 0$. & $10.3 \pm 0$. & $10.1 \pm 0$. & $10.6 \pm 0$. & $10.2 \pm 0$. & $9.9 \pm 0.2$ & $10.3 \pm 0$. & $9.3 \pm 0.2$ & $10.7 \pm 0$. & $9 \pm 0.29^{c}$ \\
\hline & 0.26 & & .27 & $7^{\mathrm{c}}$ & .26 & $29^{b^{*}}$ & 58 & $32^{\mathrm{a}^{*}}$ & 09 & $35^{\mathrm{a}^{*}}$ & 72 & $3^{b^{*}}$ & 26 & $9^{c}$ & 27 & \\
\hline RBCs & $12.7 \pm$ & $12.2 \pm 0$ & $12.5 \pm 0$ & $10.7 \pm 0$. & $12.5 \pm 0$ & $10.8 \pm 0$ & $12.5 \pm 0$. & $11.1 \pm 0$. & $12.7 \pm 0$ & $11.1 \pm 0$. & $12.8 \pm 0$. & $11.1 \pm 0$. & $12.7 \pm 0$. & $11.6 \pm 0$. & $12.8 \pm 0$. & $12.4 \pm 0$. \\
\hline million $/ \mathrm{mm}^{3}$ & 0.43 & $80^{\mathrm{a}}$ & .26 & $67^{\mathrm{a}}$ & .28 & $44^{\mathrm{a}^{*}}$ & 26 & $12^{\mathrm{a}^{*}}$ & 12 & $23^{\mathrm{a}^{*}}$ & 17 & $45^{\mathrm{a}^{*}}$ & 46 & $58^{\mathrm{a}}$ & 15 & $54^{\mathrm{a}}$ \\
\hline WBCs & $6.3 \pm 0$ & $5.2 \pm 0.3$ & $6.9 \pm 0$. & $6 \pm 0.42^{\mathrm{b}}$ & $8.4 \pm 0$ & $7.3 \pm 0.2$ & $8 \pm 0.29$ & $8.1 \pm 0.3$ & $7.3 \pm 0.3$ & $8.5 \pm 0.2$ & $7.3 \pm 0.1$ & $8.5 \pm 0.3$ & $6.8 \pm 0.2$ & $9.0 \pm 0.2$ & $6.4 \pm 0.2$ & $9.4 \pm 0.2$ \\
\hline thousand $/ \mathrm{mm}^{3}$ & .32 & $5^{\mathrm{b}}$ & 18 & & 49 & $6^{\mathrm{a}}$ & & $5^{\mathrm{a}}$ & 1 & $7^{a^{*}}$ & 5 & $2^{\mathrm{a}^{*}}$ & 0 & $7^{\mathrm{a}^{*}}$ & 4 & $0^{\mathrm{a}^{*}}$ \\
\hline
\end{tabular}

- Sho = Sham-operated $\mathrm{Hn}=$ Hydronephrosis. -Values with different superscripts letters within the same raw were statistically significant from corresponding values on day zero at $p \leq 0.05$. * within the same raw were statistically significant from corresponding values of sham-operated group at $p \leq 0.05$. 
Changes in goats with surgically- induced unilateral Hydronephrosis.

Table (2): Biochemical analysis of sham-operated goats and goats with surgically- induced unilateral hydronephrosis (Means \pm S.E)

\begin{tabular}{|c|c|c|c|c|c|c|c|c|c|c|c|c|c|c|c|c|}
\hline \multirow{2}{*}{$\begin{array}{l}\text { Days } \\
\text { Parameter } \\
\text { s }\end{array}$} & \multicolumn{2}{|c|}{0 day } & \multicolumn{2}{|c|}{$1^{\text {st }}$ day } & \multicolumn{2}{|c|}{$7^{\text {th }}$ day } & \multicolumn{2}{|c|}{$14^{\text {th }}$ day } & \multicolumn{2}{|c|}{$21^{\text {st }}$ day } & \multicolumn{2}{|c|}{$28^{\text {th }}$ day } & \multicolumn{2}{|c|}{$35^{\text {th }}$ day } & \multicolumn{2}{|c|}{$42^{\text {nd }}$ day } \\
\hline & Sh-o & $\mathrm{Hn}$ & Sh-o & Hn & Sh-o & Hn & Sh-o & Hn & Sh-o & $\mathrm{Hn}$ & Sh-o & Hn & Sh-o & Hn & Sh-o & Hn \\
\hline $\begin{array}{l}\text { Urea } \\
\mathrm{mg} / \mathrm{dl}\end{array}$ & $\begin{array}{c}28 \pm 1 . \\
53\end{array}$ & $26 \pm 4.5^{\mathrm{e}}$ & $29 \pm 0.53$ & $\begin{array}{c}29 \pm 3.79 \\
\mathrm{e}\end{array}$ & $\begin{array}{c}28.67 \pm 2 \\
.19\end{array}$ & $\begin{array}{c}45.33 \pm 2 \\
.6^{\mathrm{a}^{*}}\end{array}$ & $\begin{array}{c}28.67 \pm 0 \\
.88\end{array}$ & $\begin{array}{c}39.67 \pm 1 \\
.8^{\mathrm{b}^{*}}\end{array}$ & $\begin{array}{c}28.33 \pm 1 \\
.76\end{array}$ & $\begin{array}{c}37.33 \pm 2 \\
.7^{*}\end{array}$ & $\begin{array}{c}28.67 \pm 1 \\
.76\end{array}$ & $\begin{array}{c}35.67 \pm 2 \\
.85^{\mathrm{d}}\end{array}$ & $\begin{array}{c}28.33 \pm 0 \\
.88\end{array}$ & $\begin{array}{c}32 \pm 2.65 \\
\mathrm{e}\end{array}$ & $\begin{array}{c}28.33 \pm 1 \\
.76\end{array}$ & $\begin{array}{c}30 \pm 1.7 \\
3^{\mathrm{e}}\end{array}$ \\
\hline $\begin{array}{c}\text { Creatinine } \\
\mathrm{mg} / \mathrm{dl}\end{array}$ & $\begin{array}{l}0.70 \pm \\
0.029\end{array}$ & $\begin{array}{c}0.56 \pm 0 . \\
08^{\mathrm{d}}\end{array}$ & $\begin{array}{c}0.76 \pm 0 . \\
050\end{array}$ & $\begin{array}{c}0.85 \pm 0 . \\
128^{c}\end{array}$ & $\begin{array}{c}0.88 \pm 0 . \\
055\end{array}$ & $\begin{array}{c}1.30 \pm 0 . \\
14^{\mathrm{a}^{*}}\end{array}$ & $\begin{array}{c}0.79 \pm 0 . \\
094\end{array}$ & $\begin{array}{c}0.92 \pm 0 . \\
024^{\mathrm{b}}\end{array}$ & $\begin{array}{c}0.74 \pm 0 . \\
044\end{array}$ & $\begin{array}{c}0.76 \pm 0 . \\
034^{\mathrm{d}}\end{array}$ & $\begin{array}{c}0.69 \pm 0 . \\
035\end{array}$ & $\begin{array}{c}0.75 \pm 0 . \\
09^{\mathrm{d}}\end{array}$ & $\begin{array}{c}0.71 \pm 0 . \\
034\end{array}$ & $\begin{array}{c}0.73 \pm 0 . \\
068^{\mathrm{d}}\end{array}$ & $\begin{array}{c}0.72 \pm 0 . \\
019\end{array}$ & $\begin{array}{c}0.65 \pm 0 \\
.05^{\mathrm{d}}\end{array}$ \\
\hline $\begin{array}{l}\text { Uric acid } \\
\mathrm{mg} / \mathrm{dl}\end{array}$ & $\begin{array}{c}1.33 \pm \\
0.23\end{array}$ & $\begin{array}{c}1.01 \pm 0 . \\
09^{\mathrm{d}}\end{array}$ & $\begin{array}{c}1.34 \pm 0 . \\
23\end{array}$ & $\begin{array}{c}1.35 \pm 0 . \\
23^{\mathrm{d}}\end{array}$ & $\begin{array}{c}1.40 \pm 0 . \\
20\end{array}$ & $\begin{array}{c}2.91 \pm 0 . \\
15^{\mathrm{a}^{*}}\end{array}$ & $\begin{array}{c}1.34 \pm 0 . \\
24\end{array}$ & $\begin{array}{c}2.89 \pm 0 . \\
11^{\mathrm{a}^{*}}\end{array}$ & $\begin{array}{c}1.32 \pm 0 . \\
23\end{array}$ & $\begin{array}{c}2.68 \pm 0 . \\
29^{\mathrm{b}^{*}}\end{array}$ & $\begin{array}{c}1.34 \pm 0 . \\
22\end{array}$ & $\begin{array}{c}1.87 \pm 0 . \\
31^{\mathrm{c}}\end{array}$ & $\begin{array}{c}1.33 \pm 0 . \\
24\end{array}$ & $\begin{array}{c}1.87 \pm 0 . \\
56^{\mathrm{c}}\end{array}$ & $\begin{array}{c}1.41 \pm 0 . \\
23\end{array}$ & $\begin{array}{c}1.15 \pm 0 \\
.31^{\mathrm{d}}\end{array}$ \\
\hline $\begin{array}{l}\text { Albumin } \\
\mathrm{g} / \mathrm{dl}\end{array}$ & $\begin{array}{c}3.55 \pm \\
0.05\end{array}$ & $\begin{array}{c}3.37 \pm 0 . \\
14^{\mathrm{b}}\end{array}$ & $\begin{array}{c}3.53 \pm 0 . \\
06\end{array}$ & $\begin{array}{c}3.08 \pm 0 . \\
09^{\mathrm{b}^{*}}\end{array}$ & $\begin{array}{c}3.61 \pm 0 . \\
06\end{array}$ & $\begin{array}{c}2.88 \pm 0 . \\
05^{\mathrm{b}^{*}}\end{array}$ & $\begin{array}{c}3.52 \pm 0 . \\
04\end{array}$ & $\begin{array}{c}2.72 \pm 0 . \\
06^{\mathrm{a}^{*}}\end{array}$ & $\begin{array}{c}3.59 \pm 0 . \\
14\end{array}$ & $\begin{array}{c}2.96 \pm 0 . \\
26^{\mathrm{b}}\end{array}$ & $\begin{array}{c}3.60 \pm 0 . \\
10\end{array}$ & $\begin{array}{c}2.96 \pm 0 . \\
07^{\mathrm{b}}\end{array}$ & $\begin{array}{c}3.61 \pm 0 . \\
10\end{array}$ & $\begin{array}{c}3.02 \pm 0 . \\
26^{\mathrm{b}}\end{array}$ & $\begin{array}{c}3.53 \pm 0 . \\
11\end{array}$ & $\begin{array}{c}3.12 \pm 0 \\
.34^{\mathrm{b}}\end{array}$ \\
\hline $\begin{array}{l}\text { Total } \\
\text { proteins } \\
\mathrm{g} / \mathrm{dl}\end{array}$ & $\begin{array}{c}7.89 \pm \\
0.26\end{array}$ & $\begin{array}{c}8.03 \pm 0 . \\
17^{\mathrm{b}}\end{array}$ & $\begin{array}{c}7.89 \pm 0 . \\
26\end{array}$ & $\begin{array}{c}7.86 \pm 0 . \\
19^{\mathrm{b}}\end{array}$ & $\begin{array}{c}7.94 \pm 0 . \\
26\end{array}$ & $\begin{array}{c}7.28 \pm 0 . \\
21^{\mathrm{b}}\end{array}$ & $\begin{array}{c}7.92 \pm 0 . \\
29\end{array}$ & $\begin{array}{c}6.44 \pm 0 . \\
88^{\mathrm{a}}\end{array}$ & $\begin{array}{c}7.91 \pm 0 . \\
24\end{array}$ & $\begin{array}{c}7.03 \pm 0 . \\
46^{\mathrm{b}}\end{array}$ & $\begin{array}{c}7.82 \pm 0 . \\
26\end{array}$ & $\begin{array}{c}7.26 \pm 0 . \\
55^{\mathrm{b}}\end{array}$ & $\begin{array}{c}7.93 \pm 0 . \\
24\end{array}$ & $\begin{array}{c}7.36 \pm 0 . \\
39^{\mathrm{b}}\end{array}$ & $\begin{array}{c}7.91 \pm 0 . \\
25\end{array}$ & $\begin{array}{c}7.47 \pm 0 \\
.32^{\mathrm{b}}\end{array}$ \\
\hline $\begin{array}{l}\text { Sodium } \\
\mathrm{mEq} / \mathrm{l}\end{array}$ & $\begin{array}{c}143.3 \\
3 \pm 1.4 \\
5\end{array}$ & $\begin{array}{c}145.33 \pm \\
6.3^{\mathrm{a}}\end{array}$ & $\begin{array}{c}143.67 \pm \\
2.33\end{array}$ & $\begin{array}{c}143.67 \pm \\
3.7^{\mathrm{a}}\end{array}$ & $\begin{array}{c}144.67 \pm \\
1.20\end{array}$ & $\begin{array}{c}142.33 \pm \\
4.8^{\mathrm{a}}\end{array}$ & $\begin{array}{c}144.33 \pm \\
1.20\end{array}$ & $\begin{array}{c}142.67 \pm \\
3.3^{\mathrm{a}}\end{array}$ & $\begin{array}{c}142.67 \pm \\
0.88\end{array}$ & $\begin{array}{c}141.33 \pm \\
3.4^{\mathrm{a}}\end{array}$ & $\begin{array}{c}142 \pm 1.5 \\
3\end{array}$ & $\begin{array}{c}143.67 \pm \\
8.4^{\mathrm{a}}\end{array}$ & $\begin{array}{c}143 \pm 2.0 \\
8\end{array}$ & $\begin{array}{c}141.3 \pm 2 \\
.96^{\mathrm{a}}\end{array}$ & $\begin{array}{c}143.67 \pm \\
0.88\end{array}$ & $\begin{array}{c}144 \pm 2 . \\
65^{\mathrm{a}}\end{array}$ \\
\hline $\begin{array}{c}\text { Potassium } \\
\mathrm{mEq} / 1\end{array}$ & $\begin{array}{c}5.37 \pm \\
0.24\end{array}$ & $\begin{array}{c}5.54 \pm 0 . \\
29^{\mathrm{a}}\end{array}$ & $\begin{array}{c}5.47 \pm 0 . \\
26\end{array}$ & $\begin{array}{c}5.71 \pm 0 . \\
46^{\mathrm{a}}\end{array}$ & $\begin{array}{c}5.51 \pm 0 . \\
32\end{array}$ & $\begin{array}{c}5.73 \pm 0 . \\
54^{\mathrm{a}}\end{array}$ & $\begin{array}{c}5.37 \pm 0 . \\
27\end{array}$ & $\begin{array}{c}5.77 \pm 0 . \\
32^{\mathrm{a}}\end{array}$ & $\begin{array}{c}5.54 \pm 0 . \\
11\end{array}$ & $\begin{array}{c}5.93 \pm 0 . \\
64^{\mathrm{a}}\end{array}$ & $\begin{array}{c}5.37 \pm 0 . \\
28\end{array}$ & $\begin{array}{c}6.02 \pm 0 . \\
39^{\mathrm{a}}\end{array}$ & $\begin{array}{c}5.25 \pm 0 . \\
27\end{array}$ & $\begin{array}{c}6.17 \pm 0 . \\
11^{\mathrm{a}}\end{array}$ & $\begin{array}{c}5.33 \pm 0 . \\
26\end{array}$ & $\begin{array}{c}6.23 \pm 0 \\
.27^{\mathrm{a}}\end{array}$ \\
\hline $\begin{array}{c}\text { Chloride } \\
\mathrm{mEq} / \mathrm{l}\end{array}$ & $\begin{array}{c}120 \pm \\
1.15\end{array}$ & $\begin{array}{c}120.33 \pm \\
0.7^{\mathrm{a}}\end{array}$ & $\begin{array}{c}119 \pm 1.1 \\
5\end{array}$ & $\begin{array}{c}119.67 \pm \\
1.8^{\mathrm{a}}\end{array}$ & $\begin{array}{c}119.33 \pm \\
2.03\end{array}$ & $\begin{array}{c}119.33 \pm \\
0.9^{\mathrm{a}}\end{array}$ & $\begin{array}{c}120 \pm 1.1 \\
5\end{array}$ & $\begin{array}{c}118.67 \pm \\
1.2^{\mathrm{a}}\end{array}$ & $\begin{array}{c}119 \pm 1.1 \\
5\end{array}$ & $\begin{array}{c}119.33 \pm \\
1.5^{\mathrm{a}}\end{array}$ & $\begin{array}{c}120.33 \pm \\
2.03\end{array}$ & $\begin{array}{c}119 \pm 1.1 \\
5^{\mathrm{a}}\end{array}$ & $\begin{array}{c}120.67 \pm \\
1.45\end{array}$ & $\begin{array}{c}119.33 \pm \\
0.7^{\mathrm{a}}\end{array}$ & $\begin{array}{c}120.33 \pm \\
0.88\end{array}$ & $\begin{array}{c}120.7 \pm \\
0.8^{\mathrm{a}}\end{array}$ \\
\hline $\begin{array}{l}\text { Calcium } \\
\mathrm{mg} / \mathrm{dl}\end{array}$ & $\begin{array}{c}9.72 \pm \\
0.26\end{array}$ & $\begin{array}{c}8.96 \pm 0 . \\
10^{\mathrm{b}}\end{array}$ & $\begin{array}{c}9.67 \pm 0 . \\
26\end{array}$ & $\begin{array}{c}8.71 \pm 0 . \\
06^{\mathrm{b}}\end{array}$ & $\begin{array}{c}9.87 \pm 0 . \\
27\end{array}$ & $\begin{array}{c}7.58 \pm 0 . \\
38^{\mathrm{a}^{*}}\end{array}$ & $\begin{array}{c}9.7 \pm 0.2 \\
3\end{array}$ & $\begin{array}{c}8.19 \pm 0 . \\
70^{\mathrm{b}}\end{array}$ & $\begin{array}{c}9.84 \pm 0 . \\
35\end{array}$ & $\begin{array}{c}8.65 \pm 0 . \\
22^{\mathrm{b}}\end{array}$ & $\begin{array}{c}9.71 \pm 0 . \\
21\end{array}$ & $\begin{array}{c}8.79 \pm 0 . \\
32^{\mathrm{b}}\end{array}$ & $\begin{array}{c}9.56 \pm 0 . \\
31\end{array}$ & $\begin{array}{c}8.86 \pm 0 . \\
08^{\mathrm{b}}\end{array}$ & $\begin{array}{c}9.72 \pm 0 . \\
28\end{array}$ & $\begin{array}{c}8.94 \pm 0 \\
.12^{\mathrm{b}}\end{array}$ \\
\hline $\begin{array}{c}\text { Phosphoru } \\
\text { S }\end{array}$ & $\begin{array}{c}6.01 \pm \\
0.07\end{array}$ & $\begin{array}{c}5.63 \pm 0 . \\
27^{\mathrm{a}}\end{array}$ & $\begin{array}{c}5.99 \pm 0 . \\
11\end{array}$ & $\begin{array}{c}5.76 \pm 0 . \\
18^{\mathrm{a}}\end{array}$ & $\begin{array}{c}6.02 \pm 0 . \\
11\end{array}$ & $\begin{array}{c}5.86 \pm 0 . \\
58^{\mathrm{a}}\end{array}$ & $\begin{array}{c}5.94 \pm 0 . \\
18\end{array}$ & $\begin{array}{c}5.83 \pm 0 . \\
05^{\mathrm{a}}\end{array}$ & $\begin{array}{c}6.00 \pm 0 . \\
10\end{array}$ & $\begin{array}{c}5.88 \pm 0 . \\
29^{\mathrm{a}}\end{array}$ & $\begin{array}{c}6.05 \pm 0 . \\
13\end{array}$ & $\begin{array}{c}6.43 \pm 0 . \\
18^{\mathrm{a}}\end{array}$ & $\begin{array}{c}5.96 \pm 0 . \\
20\end{array}$ & $\begin{array}{c}5.81 \pm 0 . \\
15^{\mathrm{a}}\end{array}$ & $\begin{array}{c}6.02 \pm 0 . \\
11\end{array}$ & $\begin{array}{c}5.35 \pm 0 \\
.23^{\mathrm{a}}\end{array}$ \\
\hline
\end{tabular}

- Sho = Sham-operated $\quad \mathrm{Hn}=$ Hydronephrosis. -Values with different superscripts numbers within the same raw were statistically significant from corresponding values on day zero at $p \leq 0.05$.

-* within the same raw were statistically significant from corresponding values of sham-operated group at $p \leq 0.05$. 


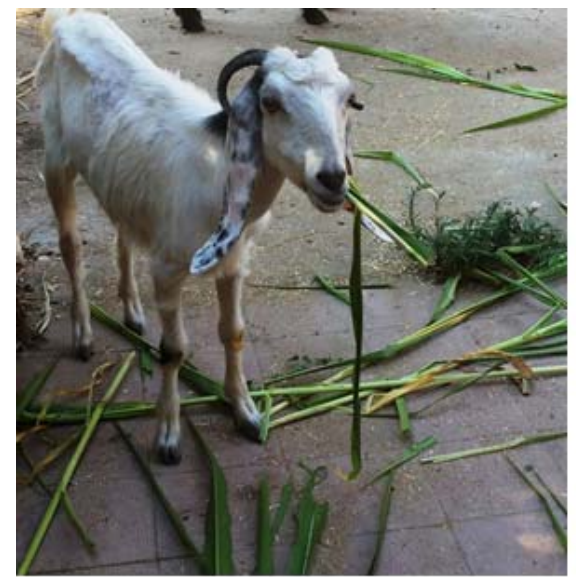

fig. 1

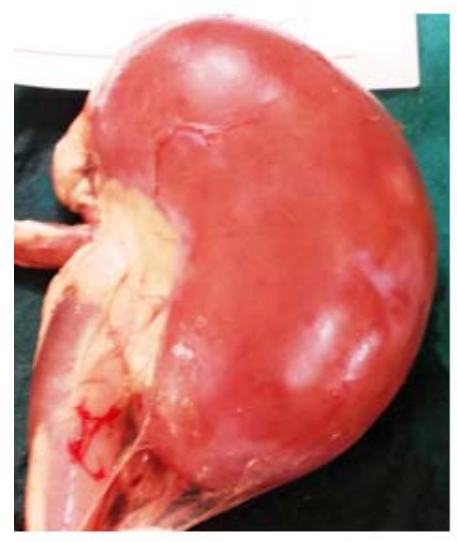

fig. $2 \mathrm{~A}$

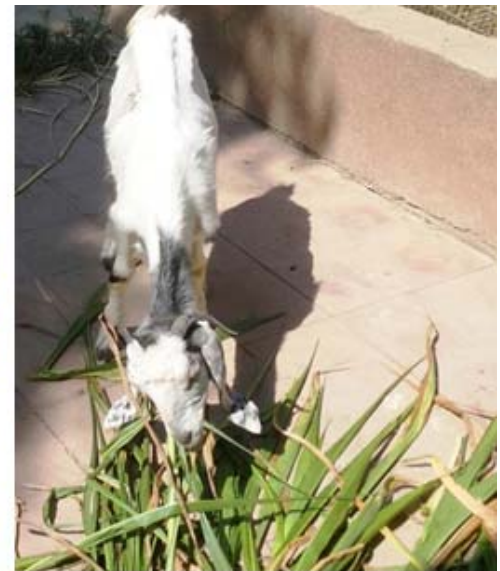

fig. 1

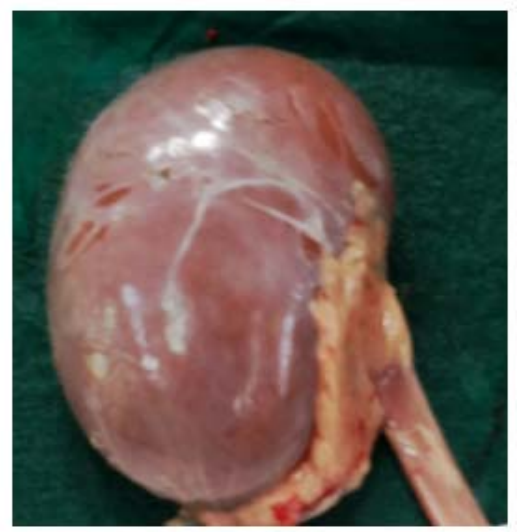

fig. 2 B

Fig.1: goat with surgically-induced unilateral hydronephrosis showed good health condition and normal appetite. Fig. 2: (A) Right kidney of goat with surgically-induced unilateral hydronephrosis showed dilatation of ureter and renal pelvis. (B) normal contralateral left kidney.

\subsection{Gross changes of kidneys of necropsied goats}

The post-mortem examination of kidneys of goats with surgically- induced unilateral hydronephrosis after 42 days post-ligation revealed dilatation of ureter and renal pelvis of the right kidney. In addition, the affected right kidney was enlarged in size with stretched capsule and filled with urine. Moreover, cut surface of the right kidney showed dilatation of renal pelvis and calyces with severe atrophy of the parenchyma due to the pressure of urine, while the left kidney appeared normal (Figure 2\&3).

\subsection{Histopathological changes}

The microscopical examination of affected kidneys collected from goats after 42 day post- ligation showed that severe degenerative changes with severe cystic dilatation of the renal tubules including proximal, distal and collecting renal tubules. The glomeruli showing severe degenerative changes and necrosis (Figure 4). Moreover, intertubular connective tissue proliferation were also detected. These proliferation leading to atrophy of the renal tubules which seen containing narrow lumen, while some of them particularly, renal cortex and most of renal tubules in the renal medulla showing cystic dilatation and periductal connective tissue proliferation (Figure 5\&6). The tubular wall become lined by flattened epithelium. The 


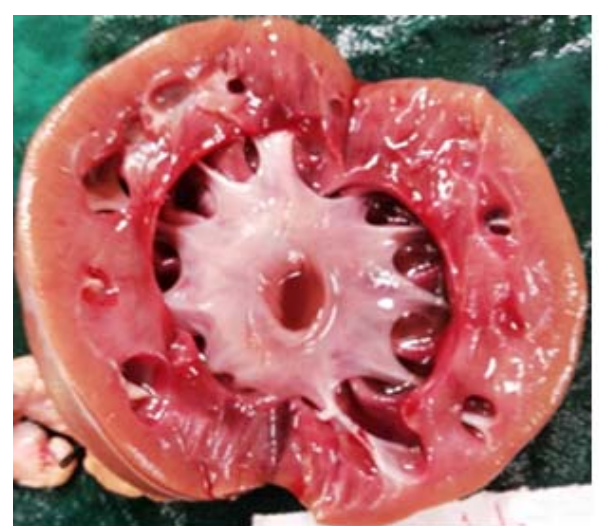

fig. $3 \mathrm{~A}$

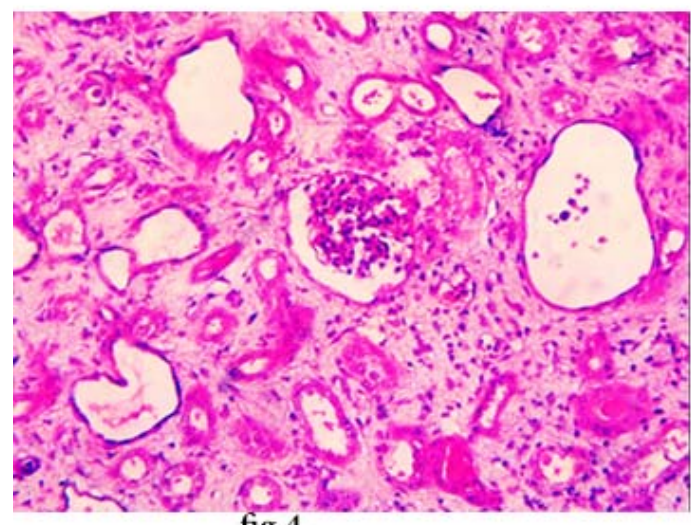

fig.4

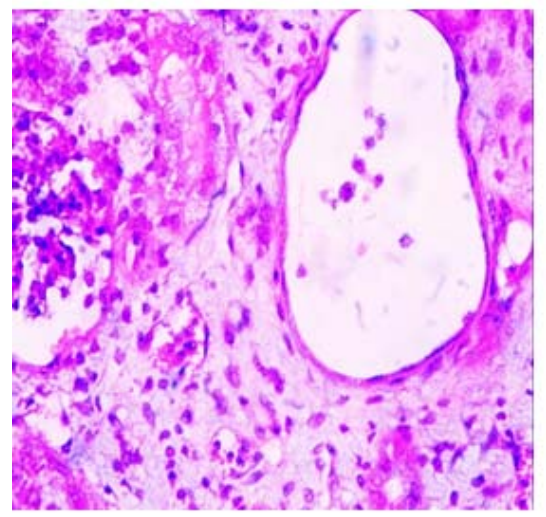

fig. 6

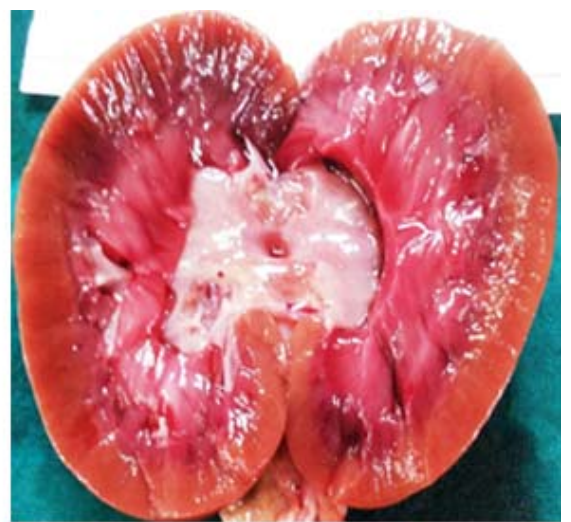

fig.3 B

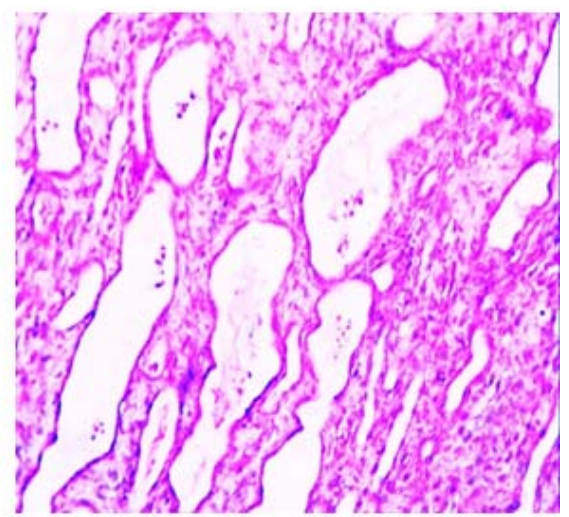

fig. 5

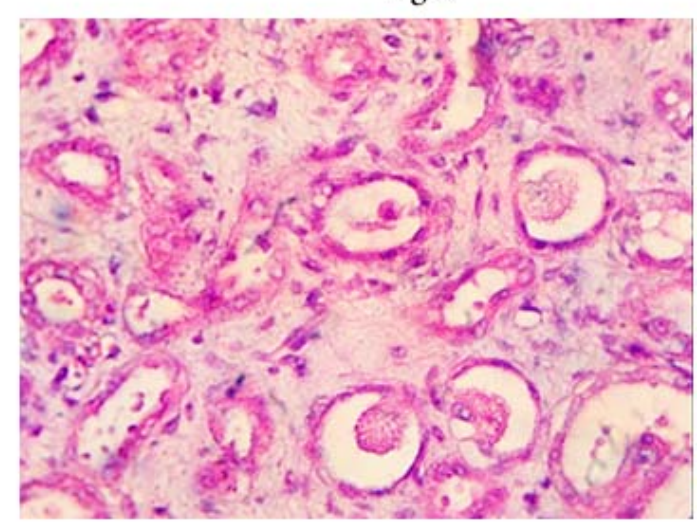

fig.7

Fig. 3: (A) Cut surface of the right kidney of goat with surgically-induced unilateral hydronephrosis showed dilatation of renal pelvis and calyces with severe atrophy of the parenchyma. (B) Normal contralateral left kidney. Fig. 4: kidney of goat at 42nd day post-ligation showing severe degenerative changes with severe cystic dilatation of renal tubules and appeared as a sac lined with flattened epithelium. (H\&E stain $\times 200)$. Fig. 5\&6: kidney of goat at 42nd day post-ligation showing intertubular fibrous connective tissue proliferation in the renal tissue. (H\&E stain $\times 400$ ). Fig. 7: kidney of goat at 42 nd day post-ligation showing Small granular or hyaline casts were in the lumen of the renal tubules. $($ H\&E stain $\times 200)$. 
intertubular blood capillaries showing mild congestion. Small granular or hyaline casts were also seen in the tubular lumen (Figure 7).

\section{DISCUSSION}

Clinical examination of goats with surgically-induced unilateral hydronephrosis showed depression, dullness, arched back and decrease in appetite than normal during the first day post obstruction. Starting from the $3^{\text {rd }}$ day the goats restored their normal appetite. The goats were returned to normal activity due to the compensatory role of contralateral kidney. These findings were nearly the same as previously mentioned by ElKammar et al. (2012).

Haematological picture of goats with surgically induced unilateral hydronephrosis revealed that the $\mathrm{PCV} \%$ was gradually decreased starting from $1^{\text {st }}$ day. On day $21^{\text {st }}$ the $\mathrm{PCV} \%$ started to increase gradually till day $42^{\text {nd }}$. The $\mathrm{Hb}$ showed a significant increase from the $7^{\text {th }}$ day post-operative then decreased gradually until returned to its normal value at the end of the study. The RBCs count showed nonsignificant decrease in the first seven days. Decrease RBCs count could be due to increase urea level in blood, which led to destruction of RBCs (Latimer, K. S., 2003). The changes in these parameters gradually returned to the normal that could be compensatory effect of the contralateral kidney. Our data were nearly agreable with that reported by El-Kammar et al. (2012).

The WBCs count was highly significantly increased on $7^{\text {th }}$ day post-operative in control sham-operated goats then decreased gradually to reach its minimal value on the $42^{\text {nd }}$ day post-operative. The WBCs count was significantly increased from $7^{\text {th }}$ day post-operative in goats with unilateral hydronephrosis and this increase remained until reached its maximal value on the $42^{\text {nd }}$ day post-operative. These findings coincided with Helal (2005). This increase in WBCs count in unilateral hydronephrosis might be attributed to surgical process and kidney damage.

The biochemical analysis showed that the serum urea level was highly significantly increased on the $7^{\text {th }}$ day post-operative then gradually decreased until reached nearly its normal level at $42^{\text {nd }}$ day of the experiment. The serum creatinine level showed significant increase on the $1^{\text {st }}$ day postoperative and the increase became highly significant on the $7^{\text {th }}$ day post-operative then gradually decreased until the end of the study. The initial increase in urea and creatinine might be due to effect of surgical ligation on kidney function before the other kidney starts its compensatory role. These results was agreed with Waltham (1999) and Frandson et al. (2009).

The serum albumin and total proteins levels were highly significant decreased on the $14^{\text {th }}$ day post-operative then gradually increased until reached normal level on the $42^{\text {nd }}$ of the study. This initial decrease in the albumin and total proteins levels could be attributed to glomerular destruction and acute phase response due to inflammation of the kidney cause decrease albumin. These data are comparable with Helal (2005) and Skotnicka et al. (2007).

The serum levels of sodium, chloride, potassium and phosphorus were insignificantly affected throughout the study. These finding in the line with Radostits et al. (2007).

The post-mortem examination of kidneys of goats with surgically- induced unilateral hydronephrosis after 42 days post-ligation revealed dilatation of ureter and renal pelvis of the right kidney. In addition, the affected right kidney was enlarged in size with stretched capsule and filled with urine. Moreover, cut surface of the right kidney showed dilatation of renal pelvis and calyces with severe atrophy of the parenchyma due to the pressure of urine, while the left kidney appeared normal. These data were coincided with those reported by Sahal et al. (2005) and Scott (2012). 
The microscopical examination of the affected kidneys after 42 day post- ligation showed that severe degenerative changes with severe cystic dilatation of the renal tubules including proximal, distal and collecting renal tubules. The glomeruli showing severe degenerative changes and necrosis. Moreover, intertubular connective tissue proliferation were also detected. These proliferation leading to atrophy of the renal tubules which seen containing narrow lumen, while some of them particularly, renal cortex and most of renal tubules in the renal medulla showing cystic dilatation and periductal connective tissue proliferation. The tubular wall become lined by flattened epithelium. The intertubular blood capillaries showing mild congestion. Small granular or hyaline casts were also seen in the tubular lumen. Our findings were agreed with Puqing et al. (1998) and McGavin et al. (2001).

\section{CONCLUSION}

Based on the obtained results of this study, it could be that unilateral hydronephrosis produced significant changes in clinical and hematobiochemical parameters during the early stage of induced Hydronephrosis. These changes were gradually restored owing to the compensatory activities of the contralateral kidney.

\section{REFERENCES}

Anderson, S.C., Cockayne, S. 1993. Clinical chemistry concepts and applications. W.B. Saunders Company, Philadelphia, London, Toronto, Montreal, Sydney, Tokyo. Pp: 429-482.

Bailey, R.A. 2008. Design of Comparative Experiments. Cambridge University Press. Pp: 116-128.

Bernard, F.F., Joseph, G.Z., Nemi, G.J. 2000. Schalm`s Veterinary Haematology. Fifth edition, USA.

Chauchan, R.S. 1995. Text of Veterinary Clinical and Laboratory Diagnosis. Joypee
Brothers Medical Publishers (P) Ltd., New Delhi, India. pp: 110- 111.

El-Kammar, M.H., Alsafy, M.A.M., Mohammed, M.I., EL-Neweshy, M.S. 2012. Ureteric obstruction and hydronephrosis in baladi goats: Topographical anatomy, ultrasonographical, hematological and pathological findings. Small Ruminant Research. 104:169-178.

Fischbach, F.T., Dunning, M.B. (2009). Manual of Laboratory and Diagnostic Tests, 8th Ed. Philadelphia: Lippincott Williams and Wilkins.

Frandson, R.D., Wilke, W.L., Fails, A.D. 2009. Anatomy and Physiology of Farm Animals, 7th Ed. Chapter 23, the Urinary System. WILEY BLACKWELL. 383-398.

Hall, L.W. 1983. Veterinary nephrology. $1^{\text {st }}$ ed. William Heinemann Medical Books Ltd. London.

Helal, M. A. 2005. Diagnosis of experimentally-induced kidney affections in dogs using ultrasonography and other methods. M.V.Sc. Thesis, 2005. Fac. Vet. Med. Benha. Univ.

Henry, R. F., Cannon, D. C., Winkelman, J.W. 1974. Clinical Chemistry Principles \& Techniques 2nd Ed. Herper \& Reo , Hagerstown MD.

Kaplan, L.A., Pesca, A. J. 1996. Clinical chemistry, first edition Mosby Company, Toronto.

Khalil, A. H. (2012). Diagnostic imaging of the uro-genital system in sheep and goat. M. V. Sc. Thesis, 2012. Fac. Vet. Med. Benha Univ.

Latimer, K.S. (2003). Duncan \& Prasse's Veterinary laboratory medicine, clinical pathology. $5^{\text {th }}$ edition. Wiley- Blackwell. pp: 3-44.

McGavin, M.D., Carlton, W.W., Zachary, J.F. 2001. The Urinary System in Thomson's Special Veterinary Pathology, 3rd ed. Mosby, Philadelphia. pp: 235-227.

Newman, S.J., Confer, A.W., Panciera, R.J. 2007. Urinary system. In pathologic Basis of Veterinary Disease (Mc Gavin M. D., Zachary J. F., eds.), 4th ed. Pp: 625680.Elsevier Mosby, St. Louis, MO.

Pagana, K.D., Pagana, T.J. 2010. Mosby's Manual of Diagnostic and Laboratory Tests $\left(4^{\text {th }}\right.$ Ed.). St. Louis: Mosby/Elsevier. 
Patton, C.J., Crouch, S.R. 1977: Enzymatic determination of urea. Anal. Chem. 49:464-469.

Puqing, Z., Gongcheng, L.U., Bo, L.I.U., Mingzhong, S. 1998. Establishment of an Animal Model of Reversible Chronic Partial Obstructive Hydronephrosis. Journal of Tongji Medical University. 18 (2): 113-114.

Radostits, O.M., Gay, C.C., Hinchcliff, K.W., Constable, P.D. 2007 Veterinary Medicine, $10^{\text {th }}$ Ed. W. B. Saunders Company Ltd, London, New York, Philadelphia, san Francisco, St Louis, Sydney.

Sahal, M., Haziroglu, R., Ozkanlar, Y., Beyaz, L. 2005. Bilateral hydronephrosis and hydroureter in a German shpherd dog. Ankara Univ. Vet. Fak Derg. 52:193-196.

Santarosa, I.A.M., Godoy, C.L.B., Pippi, N.L., Antunes, P.S.P., Rappeti, J. C.S., Krolikowski, G., Novosad, D., Gheller, V.S. 2007. Ultrasound-guided percutaneous nephrostomy in dogs. Ciência Rural. 37:762-768.

Scott, P.R. 2012. Clinical, ultrasonographic and pathological description of bladder distension with consequent hydroureters, severe hydronephrosis and perirenal fluid accumulation in two rams putatively ascribed to pelvic nerve dysfunction. Small Ruminant Research. 107: 45-48.

Skotnicka, E., Muszczyñski, Z., Dudzinska, W., Suska, M. 2007. A review of the renal system and diurnal variations of renal activity in livestock. Irish Veterinary Journal. 60(3):161-168.

Smith, M.C., Sherman, D.M. 2009. Goat medicine, 2nd Ed. Chapter 12, The Urinary System WILEY-BLACKWELL pp. 540.

Teitz, N.W. 1970. Fundam. of Clin. Chem.; W.B. Saunders Co., Philadelphia.

Tyagi, R., singh, J. 2010. Ruminant surgery. CBS Publishers \& Distributors.1st ed. pp: 6-11.

Waltham, C. 1999. The Canine Kidney. Waltham Centre for Pet Nutrition.

Wilding, P., Heath, R. 1975. Annals of Clinical Biochemistry 12:142. Cited by Wooton and Freemon (1982), pp. 79.

Young, D.S. 1990. Effect of drugs on clinical Laboratory tests. The $3^{\text {rd }}$ Ed., AACC Press, Washington, D.C. pp: 3122-3131. 